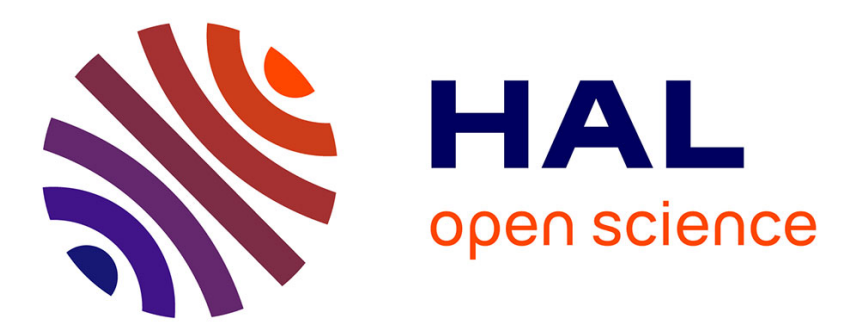

\title{
Equatorial electron density measurements in Saturn's inner magnetosphere
}

\author{
A.M. Persoon, D.A. Gurnett, W.S. Kurth, G.B. Hospodarsky, J.B. Groene, P. \\ Canu, M.K. Dougherty
}

\section{- To cite this version:}

A.M. Persoon, D.A. Gurnett, W.S. Kurth, G.B. Hospodarsky, J.B. Groene, et al.. Equatorial electron density measurements in Saturn's inner magnetosphere. Geophysical Research Letters, 2005, 32 (23), pp.L23105. 10.1029/2005GL024294 . hal-00153699

\section{HAL Id: hal-00153699 https://hal.science/hal-00153699}

Submitted on 25 Jan 2016

HAL is a multi-disciplinary open access archive for the deposit and dissemination of scientific research documents, whether they are published or not. The documents may come from teaching and research institutions in France or abroad, or from public or private research centers.
L'archive ouverte pluridisciplinaire HAL, est destinée au dépôt et à la diffusion de documents scientifiques de niveau recherche, publiés ou non, émanant des établissements d'enseignement et de recherche français ou étrangers, des laboratoires publics ou privés. 


\title{
Equatorial electron density measurements in Saturn's inner magnetosphere
}

\author{
A. M. Persoon, ${ }^{1}$ D. A. Gurnett, ${ }^{1}$ W. S. Kurth, ${ }^{1}$ G. B. Hospodarsky, ${ }^{1}$ J. B. Groene, ${ }^{1}$ \\ P. Canu, ${ }^{2}$ and M. K. Dougherty ${ }^{3}$ \\ Received 3 August 2005; revised 26 October 2005; accepted 3 November 2005; published 13 December 2005.
}

[1] Upper hybrid resonance emissions detected by the Radio and Plasma Wave Science (RPWS) instrument on the Cassini spacecraft are used to obtain electron densities on five equatorial orbits of Saturn at radial distances ranging from 3 to 9 saturnian radii $\left(\mathrm{R}_{\mathrm{S}}\right)$. The electron density profiles for these orbits show a highly repeatable radial dependence beyond $5 \mathrm{R}_{\mathrm{S}}$, decreasing with increasing radial distance approximately as $(1 / \mathrm{R})^{3.63}$. Inside $5 \mathrm{R}_{\mathrm{S}}$, the electron density profiles are highly variable. We show that these radial variations are consistent with a centrifugally-driven outward transport of plasma from a source inside $5 \mathrm{R}_{\mathrm{S}}$. Citation: Persoon, A. M., D. A. Gurnett, W. S. Kurth, G. B. Hospodarsky, J. B. Groene, P. Canu, and M. K. Dougherty (2005), Equatorial electron density measurements in Saturn's inner magnetosphere, Geophys. Res. Lett., 32, L23105, doi:10.1029/ 2005 GL024294.

\section{Introduction}

[2] The first in situ measurements of the plasma density in Saturn's inner magnetosphere were made by the plasma instruments on Pioneer 11 and Voyagers 1 and 2 during flybys of the planet in 1979, 1980 and 1981 [Frank et al., 1980; Bridge et al., 1981, 1982; Sittler et al., 1983]. From these measurements, various models were derived to describe the composition and spatial distribution of the plasma [Richardson and Sittler, 1990; Richardson, 1995, 1998; Richardson and Jurac, 2004]. The most recent plasma measurements in Saturn's magnetosphere are from the Cassini spacecraft, which was placed in orbit around Saturn on July 1, 2004. Cassini carries several instruments capable of measuring the low energy thermal plasma, one of which is the RPWS instrument [Gurnett et al., 2004, 2005]. In this paper we use RPWS measurements of the upper hybrid resonance frequency $\left(\mathrm{f}_{\mathrm{UH}}\right)$ to study the equatorial electron densities during five passes through the inner magnetosphere from October 2004 through March 2005.

\section{The Density Measurement Technique}

[3] The frequency of the $f_{U H}$ emissions has been previously used to determine electron densities in planetary magnetospheres and is now a well-established technique [Mosier et al., 1973; Warwick et al., 1979; Gurnett et al.,

\footnotetext{
${ }^{1}$ Department of Physics and Astronomy, University of Iowa, Iowa City, Iowa, USA.

${ }^{2} \mathrm{CETP} / \mathrm{CNRS} / \mathrm{IPSL}$, Velizy, France.

${ }^{3}$ Space and Atmospheric Physics, Imperial College, London, UK.
}

1981]. A frequency-time spectrogram showing an $f_{U H}$ emission band detected by the Cassini RPWS is shown in the lower panel of Figure 1. The spectrogram is from orbit 4, beginning at 22:00 Universal Time (UT) on March 8, 2005 and ending at 00:00 UT on March 10. During this time the spacecraft moves inward from a radial distance of $9.23 \mathrm{R}_{\mathrm{S}}$ at the beginning of the plot, passes through periapsis at a radial distance of $3.50 \mathrm{R}_{\mathrm{S}}$ at 11:40 UT, and then moves outward to $8.60 \mathrm{R}_{\mathrm{S}}$ at the end of the plot. Throughout this time interval the spacecraft remained very close to the equator, with a maximum latitude of 0.21 degrees. Since Saturn's magnetic dipole axis is aligned nearly parallel to its rotational axis [Connerney et al., 1983], the magnetic latitude during this pass remains very small, about one degree. The small magnetic latitude is useful because it allows us to separate radial variations from magnetic latitude variations.

[4] A very clear upper hybrid resonance band, marked $\mathrm{f}_{\mathrm{UH}}$, can be seen in the lower panel of Figure 1. The $\mathrm{f}_{\mathrm{UH}}$ emission is first observed on the inbound pass at a frequency of $28 \mathrm{kHz}$ at 23:40 UT on March 8. As the radial distance decreases, the frequency of the emission increases to a maximum of $78 \mathrm{kHz}$ at $08: 28$ UT on March 9, then decreases to a minimum of $53 \mathrm{kHz}$ near periapsis. On the outbound pass, $f_{U H}$ increases slowly to a maximum of $72 \mathrm{kHz}$ at $16: 27 \mathrm{UT}$ and then decreases to $23 \mathrm{kHz}$ at 22:50 UT. The $\mathrm{f}_{\mathrm{UH}}$ emission line is generally smooth and continuous in the region inside $6.5 \mathrm{R}_{\mathrm{S}}$, becomes progressively weaker and more sporadic beyond $6.5 \mathrm{R}_{\mathrm{S}}$, and is generally undetectable beyond $8.4 \mathrm{R}_{\mathrm{S}}$.

[5] From the basic definition of $\mathrm{f}_{\mathrm{UH}}$ [Gurnett and Bhattacharjee, 2005], the electron density, $\mathrm{n}_{\mathrm{e}}$ in $\mathrm{cm}^{-3}$, can be computed from $\mathrm{f}_{\mathrm{UH}}(\mathrm{Hz})$ using

$$
\mathrm{n}_{\mathrm{e}}=\left(\mathrm{f}_{\mathrm{UH}}^{2}-\mathrm{f}_{\mathrm{c}}^{2}\right) /(8980)^{2}
$$

where $f_{c}$ is the electron cyclotron frequency. The electron cyclotron frequency, shown by the white line marked $f_{c}$ in the lower panel of Figure 1, has been computed from onboard magnetic field measurements [Dougherty et al., 2004], using $f_{c}=28 B(H z)$ where $B$ is the magnitude of the magnetic field in nanoteslas.

[6] Two techniques can be used to determine $f_{U H}$. The passive technique consists of measuring the center frequency of the thermally excited electrostatic emissions that commonly exist at $\mathrm{f}_{\mathrm{UH}}$. The active technique consists of using the RPWS sounder transmitter to excite electrostatic oscillations at $\mathrm{f}_{\mathrm{UH}}$. In both techniques, automatic data processing routines are used to track $\mathrm{f}_{\mathrm{UH}}$ and compute the corresponding electron density. Electron densities obtained using both techniques are shown in the upper panel of 


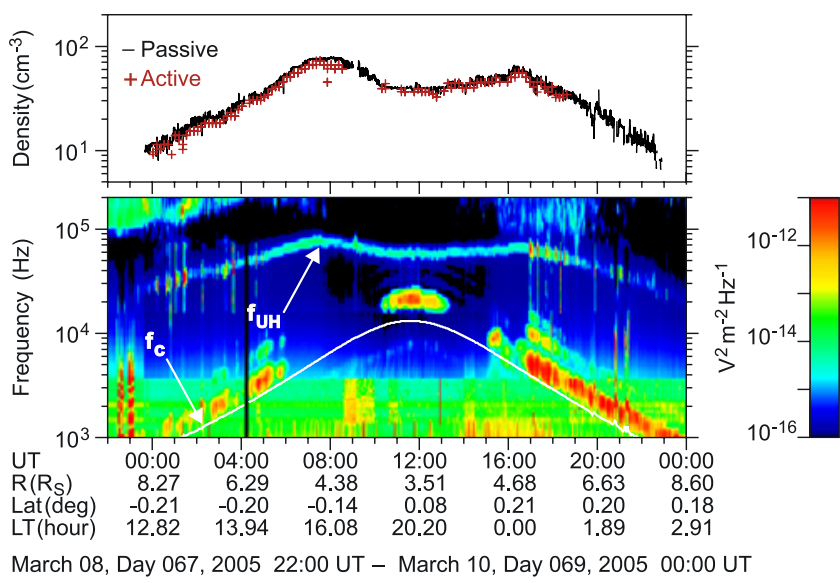

Figure 1. The frequency-time spectrogram in the lower panel shows the electric field intensities detected by the Cassini RPWS during the pass through Saturn's inner magnetosphere on March 8-10, 2005. The narrowband emission marked $\mathrm{f}_{\mathrm{UH}}$ is at the upper hybrid resonance frequency. The white line marked $\mathrm{f}_{\mathrm{c}}$ is the electron cyclotron frequency, derived from on-board measurements of the magnetic field. The upper panel shows the electron densities derived from the passive (spectrum) and active (sounder) measurements of $\mathrm{f}_{\mathrm{UH}}$.

Figure 1. The near coincidence of the two density profiles indicates very good agreement between the two techniques. When the $f_{\mathrm{UH}}$ line is strong and well-defined, the overall accuracy for computing the electron density using the passive technique is $\Delta \mathrm{n}_{\mathrm{e}} / \mathrm{n}_{\mathrm{e}} \sim 13 \%$. This accuracy degrades in regions where the signal to noise ratio is poor, i.e., less than $3 \mathrm{~dB}$. Since the active sounder measurements generally have much poorer time resolution ( 2 consecutive measurements every 10 minutes) than the passive spectrum measurements (every 8 seconds), only the passive measurements have been used in this study.

[7] The density profile in the top panel of Figure 1 shows a high degree of symmetry for the inbound and outbound portions of the orbit, reaching maximum densities at $5 R_{S}$ and decreasing smoothly with increasing radial distance beyond $5 \mathrm{R}_{\mathrm{S}}$. Inside $5 \mathrm{R}_{\mathrm{S}}$, the densities increase with increasing radial distance, indicating a change in the plasma characteristics in this region. Since the object of this study is to determine the large scale electron density profile in Saturn's magnetosphere, brief density depletions of a halforder of magnitude or more have been excluded from this study. Such short term density perturbations, believed to be due to flux tube interchange motions [André et al., 2005; Burch et al., 2005; Hill et al., 2005], are seldom observed inside $6 \mathrm{R}_{\mathrm{S}}$ and occur only a small fraction of the time $(<1 \%)$ beyond $6 \mathrm{R}_{\mathrm{S}}$.

\section{A Simple Density Model}

[8] Figure 2 shows superposed plots of the electron density as a function of radial distance for five equatorial orbits: December 14-15, 2004 (Orbit B); January 15-16, 2005 (Orbit C); February 16-17, 2005 (Orbit 3); March 89, 2005 (Orbit 4); and March 29-30, 2005 (Orbit 5). Since the focus of this study is the radial dependence of Saturn's density profile and not latitudinal variations, we have selected these five orbits because Cassini remained within $\pm 0.4 R_{S}$ of the equatorial plane inside $9 R_{S}$. In the region beyond $5 \mathrm{R}_{\mathrm{S}}$, the density profiles show a consistent repeatable radial dependence, decreasing with increasing radial distance. The scatter among the various measurements in this region is remarkably small, less than a factor of two. This is in sharp contrast to the region inside $5 R_{S}$, where the density profiles show large variations from orbit to orbit.

[9] The repeatability of the electron density profiles beyond $5 \mathrm{R}_{\mathrm{S}}$ are strongly suggestive of a nearly steadystate process. For a large rapidly rotating magnetized planet like Saturn, it has long been recognized that centrifugal forces play a dominant role in the dynamics of the magnetospheric plasma [Gledhill, 1967]. The dominance of the centrifugal force causes the co-rotating plasma to be concentrated along those parts of the magnetic field lines that are farthest from the rotational axis. For Saturn, which has its magnetic axis almost exactly aligned with its rotational axis, this means that plasma tends to accumulate near the magnetic equatorial plane.

[10] If plasma is injected at a steady rate in the inner region of the magnetosphere, then an outward radial transport of plasma must occur if the system is to achieve a steady state. This transport is widely believed to occur via an interchange instability in which plasma is exchanged between adjacent magnetic flux tubes, leading to a net flow of plasma outward from the planet. The orbit-to-orbit repeatability of the electron density profiles in Figure 2 strongly suggests that the plasma has reached a steady-state equilibrium beyond $5 R_{S}$.

[11] For a steady-state expansion, conservation of magnetic flux during the interchange process requires that $\mathrm{BA}=$ constant, where $\mathrm{B}$ is the magnetic field strength and $\mathrm{A}$ is the cross-sectional area of a flux tube. Since the magnetic field strength varies as $\mathrm{R}^{-3}$, then the cross-sectional area of the flux tube must vary as $\mathrm{R}^{3}$. In Saturn's magnetosphere, the plasma is concentrated near the magnetic equatorial plane in a disk of thickness $2 \mathrm{H}$, where $\mathrm{H}$ is the plasma scale height. The average number density in the disk $\left(\mathrm{in} \mathrm{cm}^{-3}\right.$ ) is given by

$$
\mathrm{n}_{0}=\mathrm{N} / \mathrm{A}(2 \mathrm{H}),
$$

where $\mathrm{N}$ is the total number of particles in the flux tube. Early Cassini measurements of the electron density by

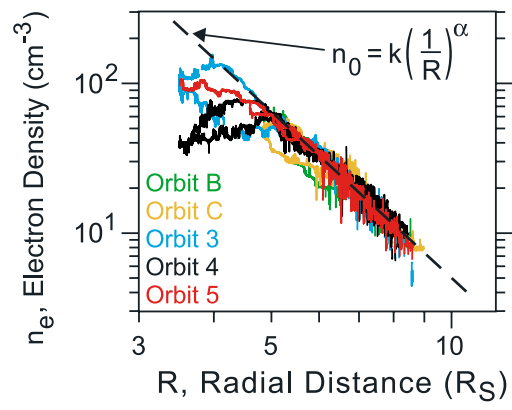

Figure 2. The electron density profiles from the RPWS measurements for five equatorial orbits as a function of radial distance from the center of the planet. The black dashed line is a power-law fit with $\alpha=3.63 \pm 0.05$ and $\mathrm{k}=$ $2.2 \times 10^{4} \mathrm{~cm}^{-3}$. 


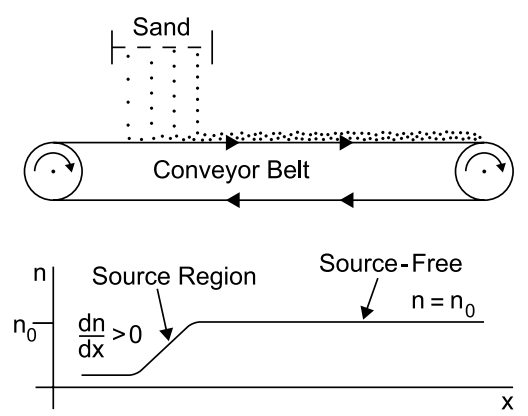

Figure 3. The upper panel shows a simple one-dimensional analogy in which sand is being deposited at a constant rate on a moving conveyor belt. The lower panel shows the resulting density profile. In the source region, the slope of the normalized density profile $\mathrm{dn} / \mathrm{dx}$ is positive. In the source-free region, the slope is zero.

Moncuquet et al. [2005] indicate that the scale height is approximately proportional to the radial distance. Substituting $\mathrm{A} \sim \mathrm{R}^{3}$ and $\mathrm{H} \sim \mathrm{R}$ into equation (2) gives $\mathrm{n}_{0} \sim \mathrm{N}(1 / \mathrm{R})^{4}$.

[12] Applying a power-law fit to the electron densities beyond $5 R_{S}$ in Figure 2 gives a radial dependence of $(1 / R)^{3.63}$. We note that this power-law radial dependence is consistent with the radial dependence inferred by Bridge et $a l$. [1982] and is close to the radial dependence expected for a source-free, steady-state radial expansion of the plasma. Whether the expansion is completely source free in the region beyond 5 RS depends critically on the radial dependence of the scale height and the flux tube content [Siscoe and Summers, 1981] and will be the subject of a future study using density measurements from orbits that have substantial latitudinal variations.

\section{Where is the Plasma Source?}

[13] Figure 2 shows that inside $5 R_{S}$, the density profiles are highly variable from orbit to orbit, even between the inbound and outbound portions of the same orbit. Densities are only available in this region for orbits 3,4 , and 5 . As they do in the region outside $5 \mathrm{R}_{\mathrm{S}}$, some densities decrease with increasing radial distance, but the radial dependence is weaker inside $5 \mathrm{R}_{\mathrm{S}}$. Some densities increase with increasing radial distance inside $5 R_{S}$. One example is the density profile in the upper panel of Figure 1 where the densities are increasing inside $5 R_{S}$, reaching a maximum value near $5 R_{S}$ on both the inbound and outbound portions of the orbit. Such a radial dependence is strongly suggestive of a plasma source located in the region inside $5 \mathrm{R}_{\mathrm{S}}$.

[14] To provide a simple one-dimensional analogy, consider a conveyor belt moving to the right with sand falling onto the conveyor belt as shown in the upper panel of Figure 3. The lower panel shows the expected particle density profile corresponding to this analogy. The particle density has been normalized by dividing by the density in the source-free region, $\mathrm{n} / \mathrm{n}_{0}$. In the region where the sand is falling, the number density of the sand particles increases with increasing distance, i.e., $\mathrm{dn} / \mathrm{dx}>0$. In the source-free region beyond where the sand is falling, however, the number density is constant, $\mathrm{n}=\mathrm{n}_{0}$.
[15] To apply the above analogy to Saturn's plasma disk, we have divided the measured densities, $n_{e}$, in Figure 2 by the best-fit, power-law model, $\mathrm{n}_{0}=2.2 \times 10^{4}(1 / \mathrm{R})^{3.63}$ in Figure 2, which represents the source-free expansion function. The results are shown in Figure 4. Beyond $5 R_{S}$, the number density ratio, $\mathrm{n}_{\mathrm{e}} / \mathrm{n}_{0}$, is very close to one, consistent with our interpretation that the plasma in this region is undergoing a nearly steady-state radial expansion with no strong evidence for localized plasma sources. This steadystate expansion of the plasma does not, however, rule out the possibility of a widely dispersed plasma source, such as the ionization of neutral gases in Saturn's magnetosphere. Inside $5 \mathrm{R}_{\mathrm{S}}$, the slopes of the normalized density profiles are consistently positive which, from the conveyor belt analogy in Figure 3, suggests that there are localized plasma sources in this region. Possible sources include Saturn's moons Enceladus and Tethys and the E ring, all of which are located in this region.

[16] An example of a density gradient in the vicinity of one of Saturn's moons occurred on March 9, 2005 at 09:08 UT when Cassini passed upstream of Enceladus at a closest approach altitude of $500 \mathrm{~km}$. The bottom panel of Figure 5 shows a one-hour spectrogram of the RPWS high resolution wideband data obtained during the Enceladus flyby. The narrowband $\mathrm{f}_{\mathrm{UH}}$ emissions are clearly evident above $60 \mathrm{kHz}$ throughout this interval, except during a 9-minute period centered on closest approach when electron plasma oscillations obscure the $\mathrm{f}_{\mathrm{UH}}$ band, making the density determination impossible.

[17] The upper panel of Figure 5 shows the high resolution density profile for this interval. Because of the high frequency resolution of the wideband receiver, the densities can be determined with an uncertainty of $\sim 1.5 \%$. The densities outside Enceladus' orbit (before 09:04 UT) are $\sim 60 \mathrm{~cm}^{-3}$. After closest approach, the densities remain high for about 12 minutes, then drop to $\sim 50 \mathrm{~cm}^{-3}$ after 09:20 UT. The density profile shows a $17 \%$ step-like density increase across the orbit of Enceladus. At closest approach, no densities can be determined so it is not possible to positively identify Enceladus as the source of the density increase. However, the plasma density increase

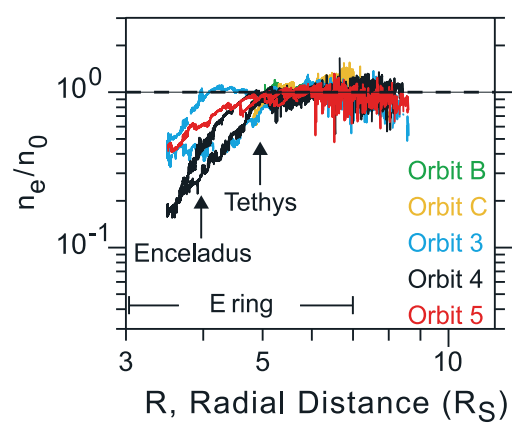

Figure 4. This log-log plot shows the normalized electron density profiles. The densities have been normalized by dividing by the best-fit power law model, shown by the dashed black line in Figure 2. Outside $5 \mathrm{R}_{\mathrm{S}}, \mathrm{n}_{\mathrm{e}} / \mathrm{n}_{0} \sim 1$, consistent with a nearly source-free, steady-state, power-law expansion. Inside $5 \mathrm{R}_{\mathrm{S}}$, the density profiles for orbits 3,4 , and 5 all exhibit a positive slope, as would be expected in a plasma source region. 


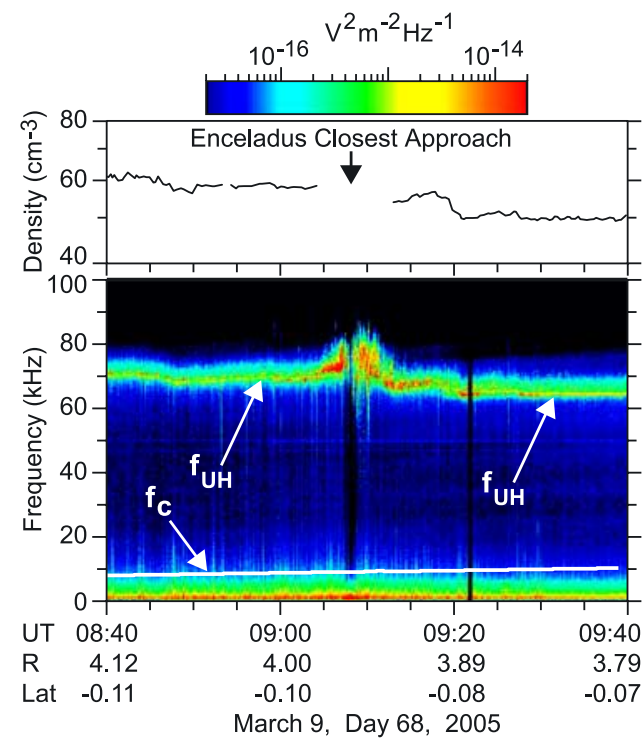

Figure 5. The lower panel shows a RPWS wideband frequency-time spectrogram during a close flyby of Saturn's moon Enceladus on March 9, 2005. There is an abrupt change in the frequency of the $\mathrm{f}_{\mathrm{UH}}$ emission band in the vicinity of Enceladus. The upper panel shows the corresponding electron density profile.

outside Enceladus' orbit is indicative of a plasma injection, either directly from gases emitted by Enceladus or from dust or ionized water vapor orbiting Saturn near the orbit of Enceladus [Jurac et al., 2002].

[18] Acknowledgment. This research was supported by NASA through JPL contract 959515 .

\section{References}

André, N., M. K. Dougherty, C. T. Russell, J. S. Leisner, and K. K. Khurana (2005), Dynamics of the Saturnian inner magnetosphere: First inferences from the Cassini magnetometers about small-scale plasma transport in the magnetosphere, Geophys. Res. Lett., 32, L14S06, doi: 10.1029/ 2005 GL022643.

Bridge, H. S., et al. (1981), Plasma observations near Saturn: Initial results from Voyager 1, Science, 212, 217-224.

Bridge, H. S., et al. (1982), Plasma observations near Saturn: Initial results from Voyager 2, Science, 215, 563-570.

Burch, J. L., J. Goldstein, T. W. Hill, D. T. Young, F. J. Crary, A. J. Coates, N. André, W. S. Kurth, and E. C. Sittler Jr. (2005), Properties of local plasma injections in Saturn's magnetosphere, Geophys. Res. Lett., 32, L14S02, doi: 10.1029/2005GL022611.

Connerney, J. E. P., et al. (1983), Currents in Saturn's magnetosphere, J. Geophys. Res., 88, 8779-8789.

Dougherty, M. K., et al. (2004), The Cassini magnetic field investigation, Space Sci. Rev., 114, 331-383.

Frank, L. A., et al. (1980), Plasmas in Saturn's magnetosphere, J. Geophys. Res., 85, 5695-5708.

Gledhill, J. A. (1967), Magnetosphere of Jupiter, Nature, 214, 155

Gurnett, D. A., and A. Bhattacharjee (2005), Introduction to Plasma Physics with Space and Laboratory Applications, p. 112, Cambridge Univ. Press, New York.

Gurnett, D. A., et al. (1981), Plasma waves near Saturn: Initial results from Voyager 1, Science, 212, 235-239.

Gurnett, D. A., et al. (2004), The Cassini radio and plasma wave investigation, Space Sci. Rev., 114, 395-463.

Gurnett, D. A., et al. (2005), Radio and plasma wave observations at Saturn from Cassini's approach and first orbit, Science, 307, $1255-1259$.

Hill, T. W., T. W. Hill, A. M. Rymer, J. L. Burch, F. J. Crary, D. T. Young, M. F. Thomsen, D. Delapp, N. André, A. J. Coates, and G. R. Lewis (2005), Evidence for rotationally driven plasma transport in Saturn's magnetosphere, Geophys. Res. Lett., 32, L14S10, doi: 10.1029/ 2005GL022620.

Jurac, S., et al. (2002), Saturn: Search for a missing water source, Geophys. Res. Lett., 29(24), 2172, doi: 10.1029/2002GL015855.

Moncuquet, M., et al. (2005), Quasi thermal noise spectroscopy in the inner magnetosphere of Saturn with Cassini/RPWS: Electron temperatures and density, Geophys. Res. Lett., 32, L20S07, doi: 10.1029/2005GL022648.

Mosier, S. R., et al. (1973), Observations of noise bands associated with the upper hybrid resonance by the Imp 6 radio astronomy experiment, J. Geophys. Res., 78, 1673-1679.

Richardson, J. D., and E. C. Sittler Jr. (1990), A plasma density model for Saturn based on Voyager observations, J. Geophys. Res., 95, 12,01912,031 .

Richardson, J. D. (1995), An extended plasma model for Saturn, Geophys. Res. Lett., 22, 1177-1180.

Richardson, J. D. (1998), Thermal plasma and neutral gas in Saturn's magnetosphere, Rev. Geophys., 36, 501-524.

Richardson, J. D., and S. Jurac (2004), A self-consistent model of plasma and neutrals at Saturn: The ion tori, Geophys. Res. Lett., 31, L24803, doi:10.1029/2004GL020959.

Siscoe, G. L., and D. Summers (1981), Centrifugally driven diffusion of iogenic plasma, J. Geophys. Res., 86, 8471-8479.

Sittler, E. C., Jr., et al. (1983), Survey of low-energy plasma electrons in Saturn's magnetosphere, J. Geophys. Res., 88, 8847-8870.

Warwick, J. W., et al. (1979), Voyager 1 planetary radio astronomy observations near Jupiter, Science, 204, 995-998.

P. Canu, CETP/CNRS/IPSL, F-92131 Velizy, France.

M. K. Dougherty, Space and Atmospheric Physics, Imperial College, London SW7 2BZ, UK.

J. B. Groene, D. A. Gurnett, G. B. Hospodarsky, W. S. Kurth, and A. M. Persoon, Department of Physics and Astronomy, University of Iowa, Iowa City, IA 52242, USA. (amp@space.physics.uiowa.edu) 